\title{
Dynamic Cognitive Self-Organized TDMA for Medium Access Control in Real-Time Vehicle to Vehicle Communications
}

\author{
Mario Manzano, ${ }^{1}$ Felipe Espinosa, ${ }^{1}$ Ángel M. Bravo-Santos, ${ }^{2}$ Enrique Santiso, ${ }^{1}$ \\ Ignacio Bravo, ${ }^{1}$ and David Garcia ${ }^{1}$ \\ ${ }^{1}$ Electronics Department, Polytechnic School, University of Alcala, Campus Universitario s/n, 28871 Alcala de Henares, Spain \\ ${ }^{2}$ Signal and Communications Theory Department, Higher Polytechnic School, Carlos III University, \\ Avenida de la Universidad 30, 28911 Leganes, Spain
}

Correspondence should be addressed to Felipe Espinosa; espinosa@depeca.uah.es

Received 21 September 2012; Accepted 23 December 2012

Academic Editor: Wuhong Wang

Copyright (C) 2013 Mario Manzano et al. This is an open access article distributed under the Creative Commons Attribution License, which permits unrestricted use, distribution, and reproduction in any medium, provided the original work is properly cited.

\begin{abstract}
The emergence of intelligent transport systems has brought out a new set of requirements on wireless communication. To cope with these requirements, several proposals are currently under discussion. In this highly mobile environment, the design of a prompt, efficient, flexible, and reliable medium access control, able to cover the specific constraints of the named real-time communications applications, is still unsolved. This paper presents the original proposal integrating Non-Cooperative Cognitive Time Division Multiple Access (NCC-TDMA) based on Cognitive Radio (CR) techniques to obtain a mechanism which complies with the requirements of real-time communications. Though the proposed MAC uses a slotted channel, it can be adapted to operate on the physical layer of different standards. The authors' analysis considers the IEEE WAVE and 802.11p as the standards of reference. The mechanism also offers other advantages, such as avoiding signalling and the adaptation capacity to channel conditions and interferences. The solution is applied to the problem of units merging a convoy. Comparison results between NCC-TDMA and Slotted-Aloha are included.
\end{abstract}

\section{Introduction}

The massive use of transportation vehicles in our society, and in particular of road transportation, has led to problems of congestion, pollution, and safety [1]. However, current advances in technology are steering the future of transport towards the intelligent transportation systems (ITS) and innovative cooperative transport alternatives $[2,3]$.

The first attempt to standardize the techniques related to ITS was the Digital Short Range Communication (DSRC) [4], developed in the United States in order to meet these communication needs. In the recent years, several organizations have led different programs and standardization efforts to cope with the specific requirements of the ITS [5]. Some of them are the industry consortia $\mathrm{C} 2 \mathrm{C}-\mathrm{CC}$ (Car to Car Communication Consortium) [6], the IEEE with the protocol suite WAVE (Wireless Access for the Vehicular Environment) [7] or the ISO (International Organization for Standardization) with
CALM (Continuous Air-Interface Long and Medium range), and the CVISs project (Cooperative Vehicle Infrastructure Systems) [8].

One of the common factors associated with these standardization activities is that the IEEE 802.11p technology is targeted to be the common V2V data link technology used for traffic safety applications [5].

As mentioned above, the IEEE proposal is the new protocol suite WAVE (Wireless Access in Vehicular Environments), which includes the description of the protocol IEEE 802.11 , belonging to the widely used 802.11 family of standards [8]. This protocol was introduced to solve the limitations inherent in nonuniversal standardization and based on the gained experience.

The aim of developing a universal standard such as WAVE, and therefore 802.11 , is to produce a standard which is generically applicable to the greatest possible number of intelligent transportation systems. Thus, the final system 
design must incorporate specific improvements to the protocol to render the latter capable of complying with the particular constraints of each application.

The 802.11p specification focuses on the physical layer and on the medium access control (MAC) sublayer, taking as its basis the best features of other specifications of the 802.11 family, widely implemented in wireless access to internet services. The medium access control protocol contains some amendments to previous specifications, such as the use of service and control channels, and the Enhanced Distributed Channel Access (EDCA) protocol [9]. Whilst these modifications improve the standar performance, they do not guarantee a total compliance with the communication requirements of certain ITS applications [10].

In order to overcome these above-mentioned limitations of the IEEE 802.11p with regard to critical ITS applications, this paper presents a medium access control mechanism (NCC-TDMA) based on a self-organized TDMA scheme which does not require signalling. To achieve this, Cognitive Radio techniques are implemented.

The rest of the paper is organized as follows. In Section 2 the limitations of 802.11p are explained in detail; Section 3 includes a summary of other proposals found in literature; Section 4 is devoted to the formal approach to the explanation of the problem and Section 5 contains the theoretical description of the solution. Finally, Section 6 provides some simulation results and Section 7 is dedicated to discussion and conclusions.

\section{Problem Description}

The physical layer and MAC sublayer of the WAVE standard, currently being developed by the IEEE with the aim of meeting ITS requirements, are described in the IEEE 802.11p specification [11].

The technology associated with the 802.11 family offers high performance in terms of wireless access to local area network (LAN) environments. This technology provides higher transmission rates than other alternatives such as WiMAX or $3 \mathrm{G}$ and is relatively cheap and easy to install and maintain. These factors make the 802.11 family an excellent option for being used as a standard in vehicle environment communications. However, in order to be included in the WAVE standard, certain amendments were necessary [12].

The physical layer of IEEE 802.11p is basically the same as that of IEEE 802.11a, due in part to the fact that both take advantage of the same frequency range $(5 \mathrm{GHz})$. The particular characteristics of vehicular environments, such as high speed nodes or rapidly changing network topology, require the inclusion of certain modifications in order to guarantee support of communication conditions in the field of ITS. IEEE 802.11p is based on applying Orthogonal Frequency Division Multiplexing (OFDM) with $10 \mathrm{MHz}$ channels, in contrast to IEEE $802.11 \mathrm{a}$ which uses $20 \mathrm{MHz}$ channels. This implies that the possible transfer rates are reduced by half, operating at $3,4.5,6,9,12,18,24$, and $27 \mathrm{Mbps}$. In addition, greater constraints have been incorporated into the receiver design to mitigate the problem of adjacent channel interference $[8]$.
As regards the medium access control sublayer, the key proposal is to simplify the connection and information exchange operations in local area wireless networks, providing effective use of adhoc communication between vehicles [8]. To this end, the infrastructure mode has been eliminated and the nodes exchange information by establishing networks in a new mode known as WAVE mode.

Using the WAVE mode eliminates the need to carry out prior network authentication and connection procedures, since in WAVE these functionalities are implemented by the upper layers of the system, as shown in Figure 1. This reduces the time and signalling required for a node to send information to network members.

On the other hand, as with the rest of the IEEE 802.11 family, the IEEE 802.11p uses Carrier Sensing Multiple Access/Collision Avoidance (CSMA/CA) as the medium access control mechanism. The only proposed improvement is the inclusion of Enhanced Distributed Channel Access (EDCA), already defined in the IEEE 802.11e amendment, whose aim is to provide the management of the quality of service. In this way the MAC mechanism of IEEE 802.11p allows to classify traffic according to the four different priorities defined in EDCA [13].

The resulting medium access control mechanism is therefore contention-based rather than deterministic, since it cannot guarantee medium access within a bounded time [10]. The improvement provided by EDCA does not guarantee deterministic access delay. As indicated in [13, 14], EDCA performance depends on the level of priority assigned to messages exchanged within the system, and the existence of a large number of high priority messages will generate an effectiveness reduction.

2.1. WAVE Multichannel Operation. Following the guidelines established from DSRC, WAVE standard includes a multichannel operation based on seven different channels. According to [15], six of them are called service channels (SCHs) and the seventh is the control channel (CCH), see Table 1. Although the EDCA strategy is applied to each of these channels, the control one is responsible for critical messages, or in terms of ITS, real-time communications. Moreover, for the control channel, the possibility of using a higher transmission power is provided in order to improve the likelihood of successfully carrying out a transmission on that channel.

In summary, the ability of WAVE to support real-time communications can be measured in the operation of the protocol on the control channel. Some of these applications are mentioned in the next section.

2.2. IEEE 802.11p Limitations. A fundamental requirement of real-time communication in the field of ITS is that the information contained in a transmission must be delivered at the appropriate time. In other words, a message must reach the receiver within a certain time and with a specified level of reliability (probability of error). Communication messages in real-time do not necessarily require high transmission rate or low delay, but it does require predictable behaviour, so that the message is received within the time limit and with 


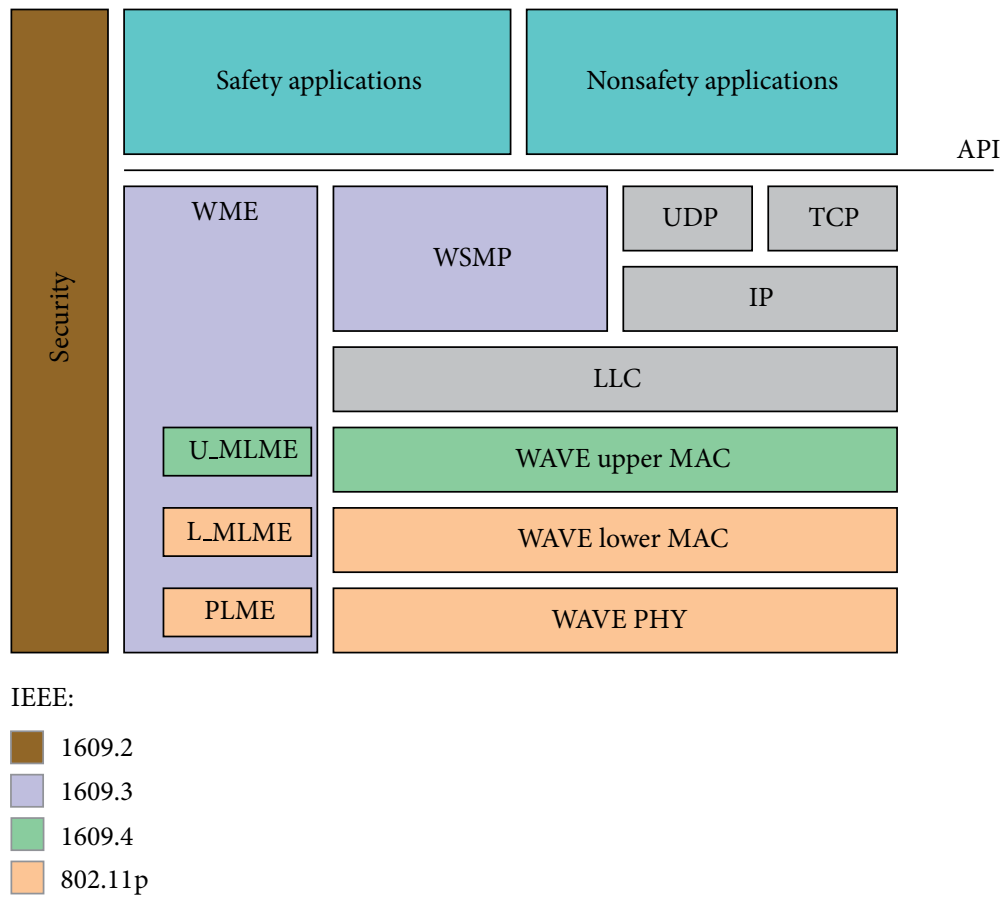

Figure 1: WAVE protocol stack.

the specified probability of error. Thus, real-time communication tasks are characterized by two important parameters: delivery time and reliability [10].

Cooperative guiding of autonomous vehicles is a clear real-time ITS application. A particular case of it is the guidance of transport units in convoy, where messages containing motion state must be delivered before global system stability is compromised [2]. This is the closest case to the system model analysed in this work.

Safety message access delay is defined as the average delay between the time at which the packet is generated and the time at which it is successfully received. According to [16], safety message access delay should be less than 200 milliseconds to allow proper driver reaction time to traffic warning signals.

The medium access control mechanism included in IEEE 802.11p is a contention-based mechanism capable of providing quality of service, but not in a deterministic way. Thus, it cannot guarantee delivery of information within an established time limit.

From the foregoing, it can be concluded that the improvement of the medium access control mechanism is required to support the special communication requirements of these ITS safety and control applications.

\section{Related Work}

Having identified the limitations of the IEEE 802.11p medium access control mechanism, in this section we will review the alternative solutions which have been proposed in the literature.
The most suitable medium access control mechanisms for implementing the specific functions required in ITS can be classified as follows [17]

(i) Contention-based protocols: the main advantage of these protocols lies in the fact that the typical network reconfiguration present in vehicular environments or Vehicular Ad Hoc Networks (VANET) does not require reconfiguration in the protocol. However, due to the random nature of medium access produced by these kinds of mechanisms, access to the medium cannot be guaranteed within a bounded time limit. These are nondeterministic mechanisms, and examples include CSMA, CSMA/CA, Aloha, and SlottedAloha.

(ii) Schedule-based or contention-free protocols: these mechanisms can provide bounded access delay, even in saturated environments, but they require reconfiguration during system operation in order to adapt to the ever-changing network topology. Examples of this are the token passing protocols (e.g., Token Ring), Time Division Multiple Access (TDMA), Frequency Division Multiple Access (FDMA), and Code Division Multiple Access (CDMA).

One of the options included in this second type protocol is TDMA. In this scheme, the shared resource is divided into time intervals which are allocated to different users for transmission, thus avoiding transmission collisions.

In a dynamic TDMA scheme, in which network topology or members are variable, schedules for access to transmission time slots are calculated continuously to reflect network 
TABLE 1: Multichannel operation in WAVE.

\begin{tabular}{lccccccc}
\hline & \multicolumn{7}{c}{ Frequency $[\mathrm{GHz}]$} \\
& 5.855 & 5.865 & 5.875 & 5.885 & 5.895 & 5.905 & 182 \\
\hline Channel number & 172 & 174 & 176 & 178 & 180 & 184 & 182 \\
Channel type & Service channel & Service channel & Service channel & Control channel & Service channel & Service channel & Service channel \\
Application & Nonsafety & Nonsafety & Traffic efficiency & Critical safety & Critical safety & Traffic efficiency Traffic efficiency \\
Radio range & C2C & Medium & Medium & All & Short & Short & Intersections \\
Tx power level & $33 \mathrm{dBm}$ & $33 \mathrm{dBm}$ & $33 \mathrm{dBm}$ & $44.8 \mathrm{dBm}$ & $23 \mathrm{dBm}$ & $23 \mathrm{dBm}$ & $40 \mathrm{dBm}$ \\
\hline
\end{tabular}

TABLE 2: Qualitative comparison of the analyzed protocols.

\begin{tabular}{|c|c|c|c|c|c|c|}
\hline \multirow{2}{*}{ Type } & CSMA/CA & Slotted-Aloha & RR-Aloha & STDMA & VeSOMAC & NCC-TDMA \\
\hline & Contention & Contention & Scheduled & Scheduled & Scheduled & Scheduled \\
\hline Topology-independent & Yes & Yes & Yes & No & No & No \\
\hline Signalling & No & No & Yes & Yes & Yes & No \\
\hline Message exchange required & No & No & Yes & Yes & Yes & No \\
\hline Possibility of collision & Yes & Yes & Yes & Yes & No & No \\
\hline $\begin{array}{l}\text { Time synchronization } \\
\text { required }\end{array}$ & No & Yes & Yes & Yes & No & Yes \\
\hline $\begin{array}{l}\text { Reason for access schedule } \\
\text { reconfiguration }\end{array}$ & $\mathrm{N} / \mathrm{A}$ & Collision & $\begin{array}{l}\text { New users, new } \\
\text { transmissions. }\end{array}$ & $\begin{array}{c}\text { Periodic } \\
\text { reconfiguration }\end{array}$ & $\begin{array}{l}\text { Collision with } \\
\text { other system } \\
\text { users }\end{array}$ & $\begin{array}{l}\text { Detection of channel } \\
\text { occupation, whether by } \\
\text { another user or due to } \\
\text { outside signal interference }\end{array}$ \\
\hline Oriented at application & No & No & No & $\begin{array}{l}\text { Yes: vehicle } \\
\text { localization and } \\
\text { movement }\end{array}$ & $\begin{array}{l}\text { Yes: vehicle } \\
\text { localization }\end{array}$ & 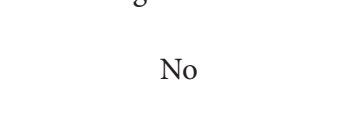 \\
\hline
\end{tabular}

changes. This schedule must be transmitted to members so that each one knows their allocated time slot. Its implementation involves time costs and requires signalling between system users in order to transmit the global schedule. Furthermore, in a centralized scheme, calculating time slot allocation schedules is carried out by a single central manager, responsible for establishing appropriate allocation within the system and transmitting this information to all the system users.

Another alternative, which is somewhat more complicated but also more suited to the special characteristics of vehicular environments, is the self-organized dynamic TDMA. In this case, there is no central unit responsible for calculating the slot allocation for the users, since allocation becomes the responsibility of the system users themselves. Consequently, the medium access control mechanism is able to contend with high levels of user mobility and network topology variations inherent in ITS.

Table 2 gives a summarized comparison of the main previous alternatives: CSMA/CA protocol included in IEEE 802.11p [7, 11], Slotted-Aloha proposals [18], RR-ALOHA [19, 20], Self-organizing Time division multiple access (STDMA [10]), and Vehicular Self Organising MAC protocol (VeSOMAC [21]), together with the basic characteristics of the authors' proposal Non-Cooperative Cognitive Time Division Multiple Access (NCC-TDMA).

The mechanism incorporated into IEEE 802.11p, together with the EDCA modification enabling the provision of quality of service, is a nondeterministic mechanism. CSMA/CA performance does not depend on network topology, since it does not need to be reconfigured when the user number or its location changes. It is a totally application-independent protocol and it does not require the use of signalling or time synchronization. The main disadvantage is that, since it does not avoid the possibility of collision, maximum delay is not bounded and situations could arise where node delivery continues indefinitely.

The Slotted-Aloha [18] is a contention-based protocol, although it also employs concepts from TDMA schemes. However, in the case of collision, selection of transmission times is based on a random process which means that maximum delay is not bounded for all cases. On the other hand, as with CSMA/CA, this mechanism does not require neither signalling nor reconfiguration when topology changes. This mechanism has been selected for comparison with NCCTDMA in Section 6.

One protocol capable of achieving dynamic channel sharing is the well-known Reservation Aloha (R-Aloha). In RAloha [22] "trial and error" transmission is used to access an available slot. If the transmission is recognized as successful then the slots reserved for that terminal in subsequent frames cannot be accessed by other terminals until the channel is released. The correct operation of R-Aloha requires a central repeater through which the terminals receive all the transmitted signals and, more important, get the same slot status information, for example, busy, free, or collided.

In order to implement a Dynamic TDMA in a distributed way, the protocol named Reliable R-Aloha (RR-Aloha [19]) 
allows the same R-Aloha procedure to be applied to vehicular communications. In RR-Aloha each terminal periodically transmits the perceived status of the slots in the preceding period (Frame Information). This information can be elaborated to get a common slot status, as in R-Aloha [22], and is used by new terminals that reach the network to get access.

RR-Aloha [19] results in a nontopology dependent protocol and it is not oriented to an specific application. On the other hand, it requires continuous transmission of signaling (Frame Information) even in a stable situation. It also requires time synchronization.

STDMA [10] is a decentralized scheme where the network members themselves are responsible for sharing the communication channel and, due to the decentralized network topology, the synchronization among the nodes is done through a global navigation satellite system, such as GPS or Galileo. The algorithm is dependent on that all nodes in the network regularly send messages containing information about their own position. The STDMA algorithm will use this position information when choosing slots in the frame. It is a protocol independent of the network topology but it is oriented to vehicle localization and movement warning applications; it requires time synchronization and, above all, channel access schedules are periodically reconfigured, even when a situation of satisfactory balance has been reached.

VeSOMAC [21] is characterized by having bounded maximum delay, at the cost of requiring periodic signalling. As with STDMA [10], it is oriented to applications based on vehicle localization although in contrast, it does not require time synchronization, it eliminates the possibility of collisions, and schedules are only reconfigured when the system detects a conflict between users over the selected slot.

The solution proposed in this paper can be classified among the schedule-based or contention-free protocols. The starting point is a self-organized dynamic TDMA which, thanks to the application of Cognitive Radio techniques (see Section 5.1. Cognitive Radio), does not require signalling messages to be sent between users in order to transmit time slot allocation schedules. This is the main difference between NCC-TDMA and others proposals found in literature.

\section{System Model}

The proposal presented in this paper has been developed on a system model applicable to the guidance of autonomous vehicles in convoy. In one such application, vehicles are grouped into convoys, each headed by a leader vehicle. These leader vehicles transmit information of the selected route to the rest of the convoy members.

In addition, the control law of each follower demands knowledge of the motion state of the preceding unit. The spread of this information is critical, or in real-time as defined by ITS, as the loss of messages between vehicles could jeopardize the stability of the convoy chain [2]. On the other hand, in the problem of cooperative guidance, there is a possibility that vehicles can leave a convoy and decide to join a different one, which incorporates variability in their sizes.

From a generic point of view, it is a system in which users (vehicles of the convoy) should convey critical information within a specified maximum deadline. The established communication system is variable with respect to the number of users (due to the ability of vehicles to join or leave each convoy) and with respect to their position (due to the mobility of the ITS vehicles).

The main difference between the work described in this paper and other ITS systems is that the number of users has been chosen within a range of lower values, closer to the convoy guided situations than other common ITS applications. Despite being a specific application in intelligent transport systems, the system model used to develop the proposal presented in this work has been generalized to make the description of the NCC-TDMA mechanism applicable to other ITS examples with real-time communication requirements.

4.1. Scenario. System users are member vehicles of an autonomous convoy that flows freely, ignoring in this paper the special conditions of start, stop, line change, and the generation of reference paths for the convoy on a real map. It is assumed that the vehicles of the transport scenario travel in convoy and they can separate from it (leaving the communication system) in the same way that other vehicles can join it (as new users).

Cooperation in the guidance system of autonomous vehicles in convoy is required in all tasks related to control as well as coordinated tracking of trajectories. However, from the point of view of CR, the system is not cooperative because the messages exchanged among users contain only information of the application, not related to the management of medium access control mechanism. This feature eliminates the need for signalling among users: vehicles never send information about the transmission slot they are using.

4.2. Channel. It is considered that communications among vehicles in a convoy are critical safe communications. Therefore, according to the definition of WAVE channels (see Table 1) only the control channel (CCH) is used.

The main requirement of real-time applications in ITS is that the exchange of messages must be made within a time limit or determined critical time $T_{c}$. In the system model, this time defines the duration of the TDMA frame. This frame is divided into different transmission slots whose duration is determined by the needs of the application.

It is assumed that (a) during each transmission slot, it is possible to transmit all the amount of information needed by each user to cover the system requirements, and (b) there are no external interferences on the communication process. Such a case should be treated by the upper layers of the protocol, which is beyond the scope of this work.

4.3. Communication. To perform the planned functions for the NCC-TDMA proposal, it is assumed that all users rely on a communication system compatible with the IEEE 802.11p standard, with the capacity to observe the medium (spectrum sensing), and with an auxiliary device able to provide time synchronization [23].

The nature of the system regarding the vehicles mobility and the possibility that a user join or separate from the 
convoy makes it impossible to solve the medium access by implementing a fixed TDMA scheme. It is not possible to allocate a slot for an indefinite period for each vehicle as they can incorporate to or separate from the convoy during the system operation. If this fixed allocation is carried out, it would not allow more incorporations than the originally planned ones, or transmission slots would remain free (without exploitation) when units leave the convoy.

This is the same reason that forces the NCC-TDMA proposal to be a fully decentralized solution where each convoy unit automatically calculates the transmission slot within the TDMA frame.

4.4. Key Aspects. In summary, the model of the communication system under study is characterized by the following restrictions.

(i) The shared medium is exploited on a TDMA scheme whose frame lasts $T_{c}$ and is divided into $r$ slots for $n$ users.

(ii) Each user must transmit its data within the limit $T_{c}$. To achieve that is necessary and sufficient the access to the medium during a transmission slot.

(iii) Once the access to the medium is gained, during the transmission period on the selected slot, it is considered that the transmission is carried out correctly.

(iv) Vehicles are equipped with a communication system capable of monitoring the environment and providing time synchronization.

(v) For the mechanism of medium access control, there is no cooperation among users. They neither exchange signaling nor have explicit knowledge of transmission slots used by the rest.

\section{NCC-TDMA Proposal}

The Non-Cooperative Cognitive Time Division Multiple Access (NCC-TDMA) proposal is aimed at guaranteeing a bounded medium access delay to ITS applications which require real-time communication. Though the proposed MAC uses a slotted channel, it can be adapted to operate on the physical layer of different standards, either by replacing the medium access control mechanism or by complementing it.

This new mechanism of medium access control is based on two of the fundamental concepts of Cognitive Radio: exploitation of the free zones of the shared medium by observing it and learning the external conditions to facilitate adequate adaption to them.

5.1. Cognitive Radio. It was during the introduction and consolidation of a great number of wireless network services and applications that Cognitive Radio (CR) emerged as a promising technology for maximizing the use of the limited radio bandwidth [24].

Cognitive Radio is an intelligent wireless communication system, which is aware of its environment and uses the methodology of understanding-by-building to learn from the environment and adapt to statistical variations in the input stimuli by effecting the corresponding changes in determined real-time parameters. The twofold aim of $\mathrm{CR}$ is to obtain a highly reliable communication whenever and wherever needed and to achieve an efficient utilization of the radio spectrum $[25,26]$.

To achieve an efficient utilization of the electromagnetic spectrum, one of the main ideas that CR exploits is the observation of the spectrum use. In a CR system, generally primary users are defined with nominal permission to use a particular frequency band, and secondary users are those that take advantage of CR to find the free radiofrequency bands between those occupied by primary users. These free zones are called spectrum holes, and searching for them, as well as using them, constitutes one of the main objectives of CR [27].

To this end, CR systems have receptors able to realize an observation of the medium "spectrum sensing" [23] and to detect its occupation by any kind of signals. The result of this observation of the occupation of the medium leads us to one of the great challenges of CR-based systems: the design of dynamic spectrum access techniques. These techniques, designed in accordance with the level of cooperation among users, range from noncooperative distributed schemes to completely centralized ones.

The NCC-TDMA proposal exploits the concept of consciousness in basing its operation on an observation of the medium occupation. Unlike conventional systems of CR, the observation of the medium does not suppose a sensing of the electromagnetic spectrum searching for free frequency bands (spectrum holes), but on an observation of the occupation of the transmission slots within the TDMA frame defined in the communication system. The other cognitive pillar of the proposal is related to the concepts of learning and adaptability. The idea exploited by the NCC-TDMA mechanism is to adapt a vector regarding the internal state of each user, to the information obtained from the observation of the occupancy of the TDMA frame. This is known as estimated allocation vector or eav, eav $\in R^{r}$ being $r$ the number of slots.

5.1.1. Observation of the Occupancy of the Medium: Transmission Schemes. A system user, after selecting a slot in the TDMA frame by running the NCC-TDMA algorithm described in next section, can access the medium generating the following situations.

(A) The selected slot is different from the slot used in the previous iteration. In this case, the user should check before transmitting whether the slot is free or not. Therefore, the total time slot is divided into

(i) a first part of observation or sensing of the medium: in which it checks if another user is occupying that time slot and

(ii) depending on the outcome of this observation it makes use of the second part of the selected slot as follows.

With the "LISTEN" action: the user listens to receive the information transmitted through the 


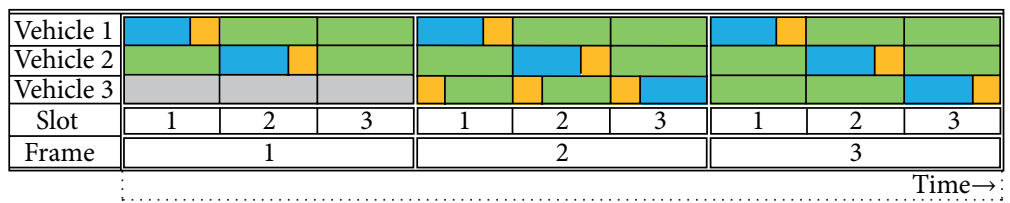

Transmission time

Medium sensing time

Listen time

Unused

FIGURE 2: Transmission schemes.

channel. This situation leads to the "SENSING + LISTEN" scheme.

With the transmission action "TX": the critical information of the user is transmitted. This situation corresponds to the "SENSING + TX" scheme.

(B) The selected slot by the algorithm is the same employed by the user in the previous iteration. Therefore, the user is considered the owner of this slot. In this case the division of the slot is conducted as follows.

(i) A primary part of transmission "TX" in which the critical information of the system is transmitted.

(ii) A second part of observing the medium "SENSING" in which it is confirmed that there are no interfering transmitters (from outside of the system) occupying the same slot of the frame. This situation results in the "TX + SENSING" scheme.

(C) Finally, the users access the medium to listen to the transmitted information in the rest of slots of the frame that were not selected by the NCC-TDMA algorithm.

Consequently, a mechanism is established in which the users that would like to transmit in new slots have to firstly check that the channel is free in these slots. The user owning the slot transmits its information in the first part of the slot, for which it will be determined that the slot is occupied by any other user intending to use that slot.

An example of the application of these transmission schemes is presented in Figure 2. It describes a simplified situation for a system of three vehicles; two of them are already operating within a convoy. Vehicle 1 transmits its critical information in the first slot of each frame, while vehicle 2 uses the second slot of each frame. Both vehicles apply the "TX + SENSING" scheme, such that after a continuous operation they transmit successfully in these slots and maintain the ownership of using them.

Vehicle number 3 appears in the system in the second frame intending to join the convoy. In this case, it is treated as a new user who should search for a free slot to carry out the transmission of its critical information. In a first trial, it selects the first slot of the second frame; therefore it observes the occupancy in the first part of the slot. Once the slot occupied by the other vehicle is detected, the second part of the slot is employed to listen to the transmitted information. During that period, a new iteration of the NCC-TDMA algorithm is done leading to the selection of the second transmission slot for a new trial. With the same result, the occupied slot is found for the second vehicle. Finally, after a new iteration of the algorithm, the third slot is chosen and is found to be free. As a result, the second part of the slot is used to transmit the critical information (the "SENSING + TX" scheme is applied in this slot).

Finally, in the third TDMA frame, vehicle number 3 can be considered as owner of this slot; as a result it will utilize the "TX + SENSING" scheme. At this moment, the system would reach an equilibrium situation. Only the appearance of new vehicles, interferences, or leaving any vehicle from the convoy could unbalance the equilibrium of the system.

5.1.2. Learning and Adaptability: Estimated Allocation Vector. The results obtained from the observation of the medium during the "SENSING" phases are saved in the eav of each vehicle. This vector has as many elements as slots in the TDMA frame, in this way each element of the vector corresponds with a slot of the frame. Each element contains scalar information about the estimation of finding that free slot. A value close to maximum indicates a high estimate to find the slot free, and a value close to minimum is associated with a high estimate to find the slot occupied.

During the operation of the system, the values of the vector eav are updated in terms of the result obtained with the observation of the medium as explained later, see Section 5.2. In this way, the different elements of the vector change such that the index of the maximum element of the vector determines the estimated free slot in each iteration. It is possible to say that eav saves the information collected at each observation and acts as a tool to choose the transmission slot in the TDMA frame.

This mechanism allows the system adapt to the changes of the environment. The changes in the occupation of the different slots of the TDMA frame are reflected in the values of the users' eav with each observation of the medium. For example, the appearance of an interfering emitter or new vehicles joining the convoy will be detected during 


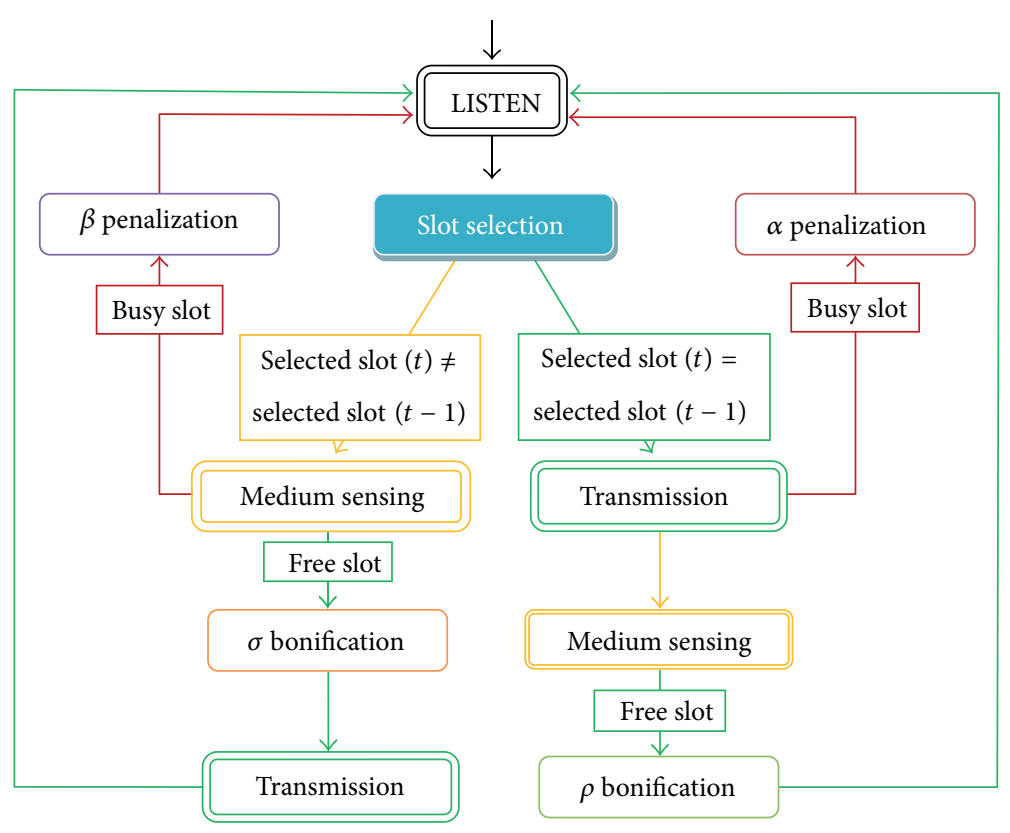

FIGURE 3: NCC-TDMA algorithm flow diagram.

the observation of the medium by each user, reducing the estimation of finding these slots free.

On the other hand, the dynamic change of users' eav causes a learning effect. The existence of interference or occupancy patterns of the TDMA frame is reflected in the medium term in the values of each user's eav. In this way, the users tend to avoid the slots that are found occupied historically.

The next section explains how the contents and the evaluation of the user's eav are parameterized.

5.2. NCC-TDMA Algorithm. The NCC-TDMA algorithm manages the operation of each user within the communication system, specifically in the medium access control. Essentially each user can carry out the next operations:

(A) medium access: in order to receive data (LISTEN), observe the occupancy of the channel (SENSING), or transmit information (TX),

(B) update its eav: starting from the result of an operation of observation of the medium (SENSING),

(C) select a slot of the TDMA frame: when it is necessary to transmit data.

Figure 3 shows the flow chart of the NCC-TDMA algorithm. The starting point of the algorithm is the "LISTEN" state. In this situation, the user is listening to the medium waiting the data reception of the rest of vehicles in the system. Once the application determines the need to transmit information, the selection of the slot is carried out.

The slot is selected by obtaining the index of the maximum value of the user's eav. In the case of starting the system operation, each user has an eav by default. For example, for a TDMA frame of 10 slots, it can be

$$
\operatorname{eav}_{\text {default }}=[10,0,7,0,6,0,3,0,0,0] \text {. }
$$

Moreover, it is necessary to know at which moment of the TDMA frame the user is found. For example, if during the execution of the algorithm, it enters into the function of slot selection at the start of the TDMA frame. The slot is determined on the complete eav, given that all the possible indices are associated in this moment with transmission slots in future time. However, if the block of slot selection is executed in the mid of the TDMA frame, only the values of the vector associated with slots in future time will be taken into account. Returning to the previous example, for a vehicle with eav, such as that of (1), executing the function of slot selection at the beginning of the frame, it will obtain the slot number 1 as a candidate (index of the maximum value of the vector). If this vehicle runs this function at an instant corresponding to the third slot, the eav on which the selection will be done agrees with

$$
\begin{aligned}
\operatorname{eav}_{\text {partial }} & =\left[\operatorname{eav}_{\text {default4 }}, \operatorname{eav}_{\text {default5 }}, \ldots, e_{\text {default9 }}, \operatorname{eav}_{\text {default10 }}\right] \\
& =[0,6,0,3,0,0,0]
\end{aligned}
$$

for which the selected slot would be number 5. As can be appreciated in (2), the partial eav coincides with the elements of (1) vector corresponding to the slots from 4 to 10 , according to the instant at which the transmission slot is being calculated.

Once the slot has been selected, it is necessary to determine whether this slot is the same as in the previous iteration 
of the algorithm. If so, this means that the user can be considered as the owner of this slot and the TX + SENSING scheme is used. On the contrary, the user should firstly be sure that the slot is free; therefore the first action to be done is the observation of the medium (SENSING).

In both cases, once the transmission has been carried out, it is returned to the initial state of listening. After the observation of the medium, the eav is updated through the bonuses or penalties application. All the bonuses and penalties consist of multiplying a parameter $(\alpha, \beta, \sigma, \rho)$ by the value of the element associated with the selected slot as follow.

Penalization $\alpha$ : given that $0<\alpha<1$, it weakens the selection of the associated slot in future iterations, it is applied when the slot considered in property is busy (the selected slot is the same as in the previous iteration).

Penalization $\beta$ : given that $0<\beta<1$, the vector element is decreased reducing the probability of selecting that slot in the next iterations. It is applied when a new slot (which is not considered in property) is busy.

Giving a Bonus $\sigma$ : given that $1<\sigma$, the vector element increases. It takes place when the observation of the medium indicates that a new free slot has been found, and therefore, it is expected to remain free in future iterations. This slot is given a bonus to enhance its choice in the next cycles.

Giving a Bonus $\rho$ : in the same mode like $\sigma, 1<\rho$. In this case, the vector element is given a bonus when the slot in which transmission took place is free and is considered in property.

In case of not transmitting in the selected slot (after applying $\alpha$ or $\beta$ penalties), a new iteration of the algorithm is triggered. In case of having a successful transmition, after applying $\sigma$ or $\rho$ bonuses, the user remains in the LISTEN state until the next TDMA frame.

\subsubsection{Characteristic Parameters of the NCC-TDMA Algorithm.} The NCC-TDMA algorithm is parameterized so that it is possible to control the behavior of the mechanism. That is, it is possible to modify the mode in which the observation on the medium occupation influences the development of the user's eav. A rapid evolution of the vector causes the users to change their slot often before minor variations of the medium. A slow evolution ensures that the users tend to stay in the same slots.

All parameters control or limit the evolution of the values of the eav components. The number of iterations required for convergence of the system to an equilibrium situation in the medium access depends on the primary and secondary parameters.

The primary parameters are those of bonuses $(\rho$ y $\sigma$ ) and penalties $(\alpha$ y $\beta)$ already described in the previous section.

On the other hand, the following secondary parameters or restrictions on eav are defined.

(i) Maximum value eav $\max _{\operatorname{ma}}$ : limits the upper value of any element of the vector. Giving a bonus to an element is not applied if it has already reached the maximum value. (ii) Minimum value eav $\min _{\text {min }}$ : it is assumed that the minimum value is zero. Hence, the values of the elements of the vector vary between 0 and eav $_{\max }$.

(iii) Constant total sum eav sum : the sum of all elements of eav remains constant during the iterations of the algorithm. This implies that when applying a bonus on an element, the increment should be subtracted from other nonnull elements of the vector. Similarly, the decrement after applying a penalty should be distributed among other elements of the vector until the total sum is eav sum $_{\text {. }}$.

(iv) Number of nonnull elements eav NonZero: a fixed number of vector elements whose value is not zero must be maintained. Thus, there are always candidate elements of the vector to be selected during the iteration of the algorithm.

For a better understanding of the operation of the algorithm and the function of each of the defined parameters, different examples of applications are included in the results section.

5.3. Equilibrium. Given that the present work is limited to the situation in which the maximum number of users transmitting at the same TDMA frame has to be less than or equal to the number of slots in the TDMA frame, possible solutions for media access, or equilibrium situations, can be described mathematically as detailed below.

As already indicated, the eav of each user contains the estimated information of occupancy of the slots of the TDMA frame. This vector has as many elements as there are slots in the TDMA frame, so that each vector element is associated with each one of the slots in the frame. According to this information, the algorithm selects a slot to access the medium in each iteration.

Similarly, you can define an allocation vector with as many elements as slots in the TDMA frame so that each element of the vector is associated to each slot of the frame. Each element will have a value of 1 for a slot occupied by the user and 0 for the remaining values. By combining all these user allocation vectors, the system allocation matrix is obtained.

For example, in a system with 4 users and 10 transmission slots, the allocation vector for user 1 occupying the third transmission slot is

$$
a_{1}=[0010000000] .
$$

Thus, the allocation matrix takes the following form:

$$
A=\left(\begin{array}{l}
0010000000 \\
0100000000 \\
0001000000 \\
1000000000
\end{array}\right) .
$$

The matrix shown in (4) corresponds to a system in which users 1, 2, 3, and 4 transmit in slots 3, 2, 4, and 1, respectively. In other words, the matrix reflects an organized TDMA system in which each user is allocated during an exclusive transmission slot. 
When this approach is applied to a system of $n$ users and $r$ slots (where $r \geq n$ ), resolving transmission schedule is reduced to finding how to order the matrix in such a way that the unit values of each user's allocation vector are located in different matrix columns. This situation is described by what are known as permutation matrices.

A permutation matrix is a square matrix where all its $n \times n$ elements are equal to 0 , except one, which should be equal to 1 , in each row and column. According to this definition, $n$ ! different permutation matrices are possible.

If this definition is applied to the problem under study, the outcome is a situation where, in an organized system of $n$ users and r slots, each of the users transmits using a slot which is not occupied by any other system user.

The strict definition of a permutation matrix is limited to a square matrix corresponding to a system with the same number of available slots as users. This situation is the most restrictive for the medium access control mechanism described in this section, but it is not the only one since a system where the number of available transmission slots is greater than the number of users can also be applied. Thus, in this paper we extend the definition of a permutation matrix to include any matrix of $n \times r$ elements (where $r \geq n$ ) in which each of the rows has only one value equal to 1 , located in a column in which there is no other value equal to 1 . According to this extended definition, the matrix in (4) is a permutation matrix.

In the case of $n$ users in a system with $r$ slots, the number of possible matrix permutations is given by the following expression:

$$
P(r, n)=\frac{r !}{(r-n) !}, \quad \forall r \geq n
$$

Taking the description of the solution to the problem one step further, the aim is to reorder the system allocation matrix so that it becomes a permutation matrix.

\section{Results}

In order to quantify the NCC-TDMA mechanism performance, a simulation experiment has been designed taking into account the special scenario of autonomous vehicles guidance and platooning manoeuvring. For such scenario, a reduced number of users have to be considered, in a different way than typical ITS experiments where lots of users are involved. Results shown in this paper are a new updated version of the results included in the authors' previous work [28].

The performed test considers the initial state of a group of transport units trying to form a convoy. Given a set of users with a number of transmission slots available, an initial situation was proposed in which each of the users had randomly selected a slot. The random selection of each user initial state was performed in order to start the test with a conflict situation between two or more users. In that way the algorithms under study are forced to find an equilibrium solution without conflicts among users. The number of algorithm iterations (number of time slots required to find

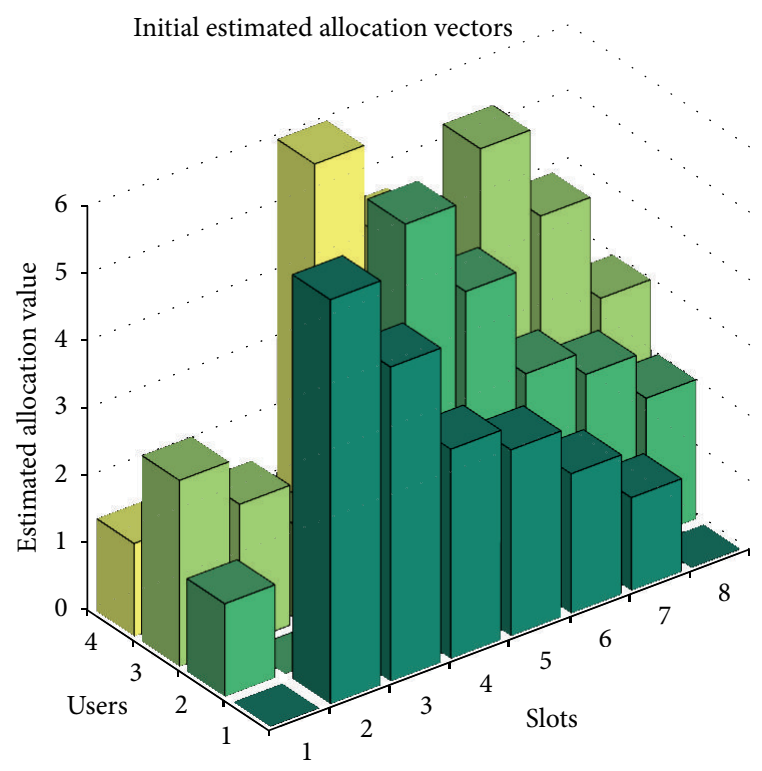

FIGURE 4: Initial estimated allocation vectors of the example with 4 users and 8 slots.

TABLE 3: Characteristic parameters for the experiment with NCCTDMA.

\begin{tabular}{lccccccc}
\hline eav $_{\max }$ & $\mathrm{eav}_{\min }$ & $\mathrm{eav}_{\text {sum }}$ & $\mathrm{eav}_{\text {NonZero }}$ & $\rho$ & $\sigma$ & $\alpha$ & $\beta$ \\
\hline 6 & 0 & 20 & 6 & 1.2 & 1.2 & 0.2 & 0.2 \\
\hline
\end{tabular}

the equilibrium) has been counted to measure the algorithm performance. In order to find the equilibrium, the algorithm has to reorder the transmission schedule so that each user could select a slot which had not been selected by another.

With the aim of simplifying the experiment, the application example considers 4 users and 8 slots. Firstly, a graphic illustration of how the NCC-TDMA algorithm operates is provided. Figure 4 shows the randomly generated eav's which values were obtained for a simulation with 4 users and 8 slots. These initial vectors were generated according to the parameters shown in Table 3. The slots selected by each user, at the initial iteration, are shown in Figure 5 (maximum values of each vector).

Focusing on the algorithm efficiency to reorder the schedule, when conflict arose among users, the TX + SENSING scheme is assigned to low user identifiers and the SENSING + TX scheme in other cases.

Since initial strategy selection was random, 2 vehicles were detected as being in conflict at the start of the assay. These were vehicles 2 and 4, whose maximum values of its estimated allocation vectors were located in slot 4 . To solve the conflict, NCC-TDMA waits until the initial time of the conflict slot; at this moment the algorithm of the user in conflict changes its selection by updating its estimated allocation vector by bonus and penalties. The new slot selection is performed until the equilibrium is reached. For the basic test described here to show the mechanism behaviour, user 4 reallocates its selection to use slot number 5 after 5 iterations, as shown in Figures 6 and 7. 


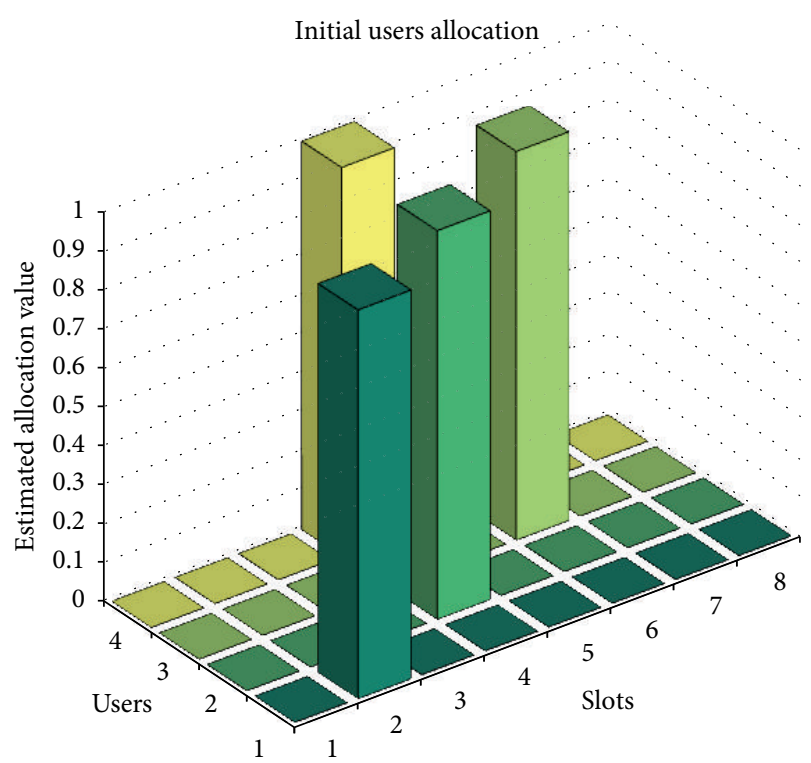

FIgURE 5: Initial allocation for the example under test.

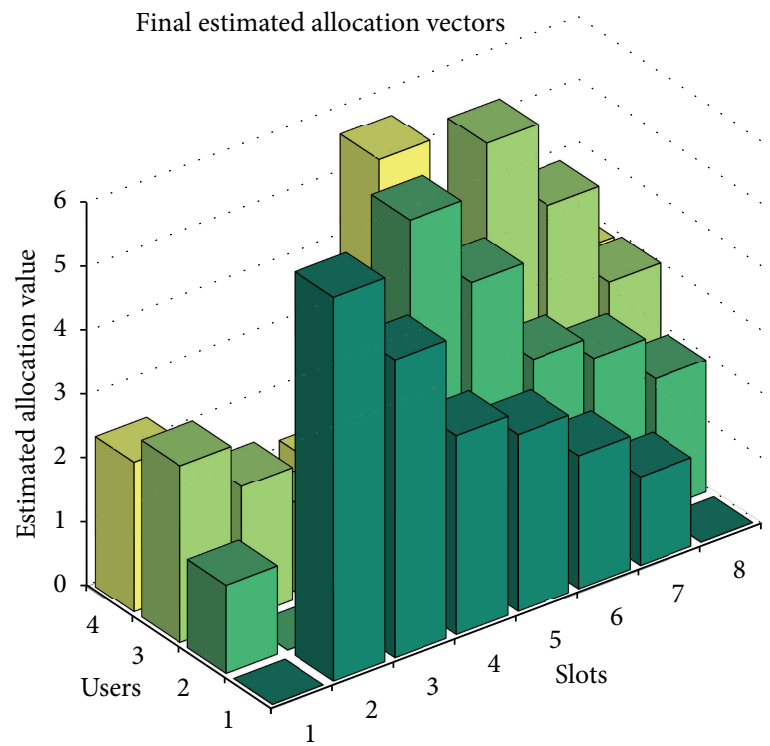

FIGURE 6: Final estimated allocation vectors of the example with 4 users and 8 slots.

In all the cases, following successful transmission, the eav value associated with the corresponding slot was awarded an incentive to reinforce the same decision in future iterations. In this way, once equilibrium had been reached, the eav values for each user's slot selection were reinforced maintaining the equilibrium state.

6.1. Comparison with Slotted-Aloha. With the objective of comparing the authors' proposal with another alternative medium access control protocol, the same simulation experiment was carried out using the Slotted-Aloha mechanism [18].

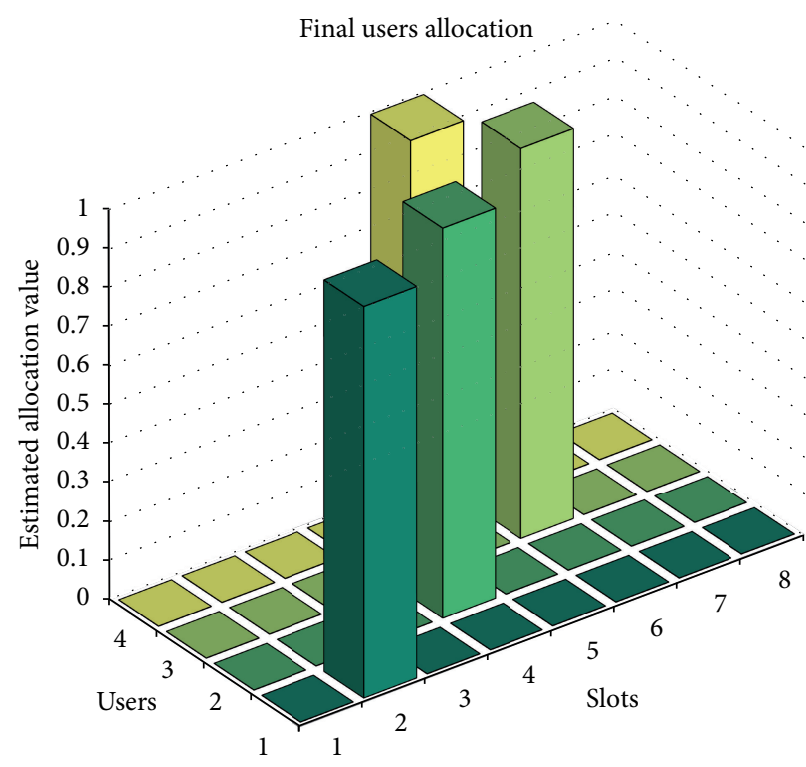

FIgURE 7: Final allocation for the example under test.

Mean iterations to reach equilibrium using Slotted-Aloha

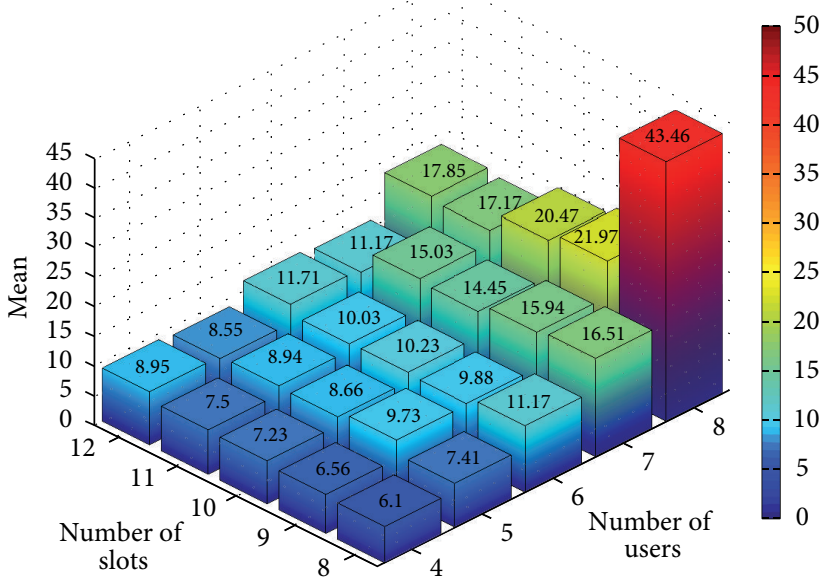

FIGURE 8: Results of the repeated experiment using Slotted-Aloha.

The Slotted-Aloha protocol was selected for two reasons. Firstly, because it is one of the most widely studied protocols in the literature, and secondly because we considered it more instructive to compare the NCC-TDMA with a generic MAC mechanism which is not oriented to the application and does not use signalling, as it is the case of STDMA [10] and VeSOMAC [21].

In the Slotted-Aloha MAC mechanism [18], medium access is obtained using TDMA time slots. When a user needs to transmit, this is attempted at the beginning of the following time slot. If this slot is occupied, a random number is generated to determine the number of time slots the user must wait for attempting a new transmission. In case of collisions, the involved users must wait for a random number of slots, implying a certain level of probability that the collision will be repeated. 
Mean iterations to reach equilibrium using NCC-TDMA

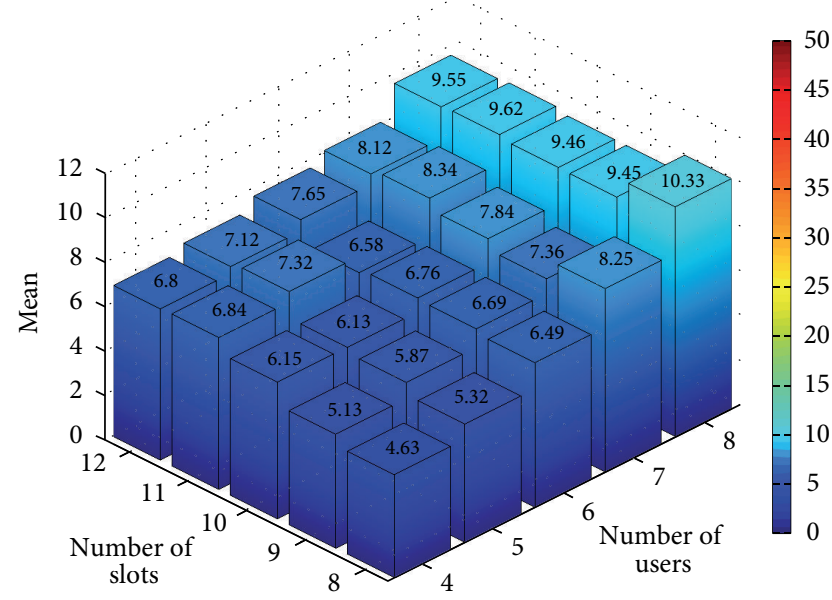

FIGURE 9: Results of the repeated experiment using NCC-TDMA.

The results shown in Figures 8 and 9 correspond to the mean number of iterations necessary for each mechanism to achieve the equilibrium state where each user has a free slot, coming from a randomly generated access schedule.

The mean value was obtained after 100 repetitions and for simulations using from 4 to 8 users and from 8 to 12 slots. The characteristic parameters shown in Table 3 were applied to NCC-TDMA. The most restrictive simulation was the one involving 8 users and 8 slots. As can be observed, for the same number of slots, the greater number of users the greater number of iterations needed, due to the possibility of conflict among users increases. The number of iterations is evaluated as the number of time slots until the equilibrium is reached. In fact, this increment in the number of iterations is caused by the position of the conflict, not for the algorithm performance requirements. In the most restrictive case, the NCC-TDMA mechanism solves the medium access order of users applying less iterations than the Slotted-Aloha mechanism. The mean number of iterations for NCC-TDMA is 10.33 and the mean number of iterations for Slotted-Aloha is 43.46 .

\section{Discussion and Conclusion}

This work describes a medium access control mechanism complying with real-time requirements for ITS applications. Special mention deserves V2V case of platooning guidance and manoeuvring where the loss of critical information commits the global stability of the convoy formation.

The main contribution of the NCC-TDMA proposal is a schedule-based mechanism, which, on one hand, eliminates the possibility of medium access collision and, on the other hand, does not require signalling. Thanks to Cognitive Radio techniques as spectrum sensing, learning, and adaptability, it is a generic protocol capable of detecting conflicts among system users or due to outside signal interference.

As in other systems based on self-organized TDMA, each user is responsible for determining the medium access schedule employed by the system. Furthermore, with the
NCC-TDMA, users have the necessary information without needing any more than the information they themselves can obtain from observation of the channel. Consequently, they are capable of detecting the occupation of a slot, whether this is occupied by another user or by an interfering signal.

The result is a dynamic mechanism which adapts to environmental conditions, however, variable these may be. Moreover, the mechanism is capable of learning from these conditions, for instance, avoiding certain transmission slots which are sporadically occupied by interfering signals.

For guidance of autonomous vehicles in convoy, the medium access controls is of vital relevance in split and merge manoeuvres. In these cases, the mechanism of medium access control must deal with new vehicles joining the convoy, keeping a satisfactory transmission for member vehicles. Finally, it must be highlighted the fact that the NCC-TDMA mechanism is capable of acting as a stand-alone medium access control protocol, or as a complement to the protocol incorporated into the WAVE standard. However, this first version does require time synchronization for each user.

To summarize, it can be concluded that the medium access control mechanism described by authors is characterized by the following.

(i) It is based on a self-organized dynamic TDMA scheme.

(ii) It does not require the exchange of signalling among users.

(iii) It does not require compulsory exchange of frames among users if a user does not have any application information to transmit.

(iv) Once a stable situation has been reached, the mechanism remains in this state until it detects a new event, which may include channel occupation by another user in the system, and channel occupation by an outside interfering signal.

(v) The system adapts to environment variations and is capable of learning from repeated situations.

However, there are some situations in which the current version of NCC-TDMA is not able to cope with by its own. The case of two or more users exploring a free slot at the same time and finding it as free will provoke a conflict in the next iteration as both users will transmit in the same slot. Same problem will happen according to the well-known hidden terminal problem. For both cases, authors are working on NCC-TDMA review where these problems are going to be tackled in higher layers of the protocol stack where the NCCTDMA offers alternatives to update the estimated allocation vector. On top of that, two main work lines have been identified as future work.

(i) The NCC-TDMA algorithm will be tested against disturbances and uncertainties over the specific scenario of $\mathrm{V} 2 \mathrm{~V}$ communication in generic ITS environments.

(ii) The NCC-TDMA algorithm efficiency will be compared with other alternatives for $\mathrm{V} 2 \mathrm{~V}$ communication that uses signalling as RR-Aloha. 


\section{Acknowledgments}

This work has been supported by the Spanish Ministry of Science and Innovation through the VISNU (Ref. TIN200908984) and the ESPIRA (Ref. 2010-2013) Projects.

\section{References}

[1] J. Jakubiak and Y. Koucheryavy, "State of the art and research challenges for VANETs," in Proceedings of the 5th IEEE Consumer Communications and Networking Conference (CCNC '08), pp. 912-916, January 2008.

[2] C. Santos, F. Espinosa, D. Pizarro, F. Valdés, E. Santiso, and I. Díaz, "Fuzzy decentralized control for guidance of a convoy of robots in non-linear trajectories," in Proceedings of the IEEE Conference on Emerging Technologies and Factory Automation (ETFA '10), pp. 1-8, 2010.

[3] W. Wang, Y. Mao, J. Jin et al., "Driver's various information process and multi-ruled decision-making mechanism: a fundamental of intelligent driving shaping model," International Journal of Computational Intelligence Systems, vol. 4, no. 3, pp. 297-305, 2011.

[4] Y. L. Morgan, "Notes on DSRC \& WAVE standards suite: its architecture, design, and characteristics," IEEE Communications Surveys and Tutorials, vol. 12, no. 4, pp. 504-518, 2010.

[5] G. Karagiannis, O. Altintas, E. Ekici et al., "Vehicular networking: a survey and tutorial on requirements, architectures, challenges, standards and solutions," IEEE Communications Surveys and Tutorials, vol. 13, no. 4, pp. 584-616, 2011.

[6] CAR 2 CAR Communication Consortium Manifesto, 2007, http://elib.dlr.de/48380/1/C2C-CC_manifesto_vl.1.pdf.

[7] D. Jiang and L. Delgrossi, "IEEE 802.11p: towards an international standard for wireless access in vehicular environments," in Proceedings of the 67th IEEE Vehicular Technology Conference (VTC '08), pp. 2036-2040, May 2008.

[8] T. Ernst, V. Nebehaj, and R. Søråsen, "CVIS: CALM proof of concept preliminary results," in Proceedings of the 9th International Conference on Intelligent Transport Systems Telecommunications (ITST '09), pp. 80-85, October 2009.

[9] N. Ferreira, J. A. Fonseca, and J. S. Gomes, "On the ad cy of 802.11p MAC protocols to support safety services in ITS," in Proceedings of the IEEE International Conference on Emerging Technologies and Factory Automation, pp. 1189-1192, 2008.

[10] K. Bilstrup, E. Uhlemann, E. G. Ström, and U. Bilstrup, “On the ability of the 802.11p MAC method and STDMA to support realtime vehicle-to-vehicle communication," EURASIP Journal on Wireless Communications and Networking, vol. 2009, Article ID 902414, pp. 1687-1472, 2009.

[11] IEEE 802.11p, "Standard for Information technologytelecommunications and information exchange between systems-local and metropolitan area networks-specific requirements-part 11: Wireless LAN Medium Access Control (MAC) and Physical Layer (PHY) Specifications. Amendment 6: Wireless Access in Vehicular Environment".

[12] R. A. Saeed, A. B. HjNaemat, A. B. Aris, I. M. Khamis, and M. K. Bin-Awang, "Evaluation of the IEEE 802.11p-based TDMA MAC method for road side-to-vehicle communications," International Journal of Network and Mobile Technologies, vol. 1, no. 2, pp. 81-87, 2010.

[13] S. Sehrawat, R. P. Bora, and D. Harihar, "Performance analysis of QoS supported by Enhanced Distributed Channel Access
(EDCA) mechanism in IEEE 802.11e," IAENG International Journal of Computer Science, vol. 33, article 1, 2007.

[14] S. Eichler, "Performance evaluation of the IEEE 802.11p WAVE communication standard," in Proceedings of the 66th IEEE Vehicular Technology Conference (VTC '07), pp. 2199-2203, 2007.

[15] S. A. Mohammad, A. Rasheed, and A. Qayyum, "VANET architectures and protocols stacks: a survey," in Communication Technologies for Vehicles, vol. 6596 of Lecture Notes in Computer Science, pp. 95-105, Springer, 2011.

[16] K. Fawaz, A. Ghandour, M. Olleik, and H. Artail, "Improving reliability of safety applications in vehicle ad hoc networks through the implementation of a cognitive network," in Proceedings of the 17th IEEE International Conference on Telecommunications (ICT '10), pp. 798-805, April 2010.

[17] S. Kumar, V. S. Raghavan, and J. Deng, "Medium access control protocols for ad hoc wireless networks: a survey," Ad Hoc Networks, vol. 4, no. 3, pp. 326-358, 2006.

[18] L. Roberts, "Aloha packet system with and without slots and capture," ACM SIGCOMM Computer Communication Review, vol. 5, no. 2, pp. 28-42, 1975.

[19] F. Borgonovo, A. Capone, M. Cesana, and L. Fratta, "ADHOC MAC: new MAC architecture for ad hoc networks providing efficient and reliable point-to-point and broadcast services," Wireless Networks, vol. 10, no. 4, pp. 359-366, 2004.

[20] F. Borgonovo, A. Capone, M. Cesana, and L. Fratta, "RRALOHA, a Reliable R-ALOHA broadcast channel for ad-hoc inter-vehicle communication networks," Dipartimento Elettronica e Informazione, Politecnico di Milano, 2002medhoc.pdf 2002medhoc.pdf.

[21] F. Yu and S. Biswas, "Self-configuring TDMA protocols for enhancing vehicle safety with DSRC based vehicle-to-vehicle communications," IEEE Journal on Selected Areas in Communications, vol. 25, no. 8, pp. 1526-1537, 2007.

[22] W. Crowther, R. Rettberg, D. Waldem, S. Ornstein, and F. Heart, "A system for broadcast communications: reservation ALOHA," in Proceedings of the 6th Hawaii International Conference on System Sciences, January 1973.

[23] D. Cabric, S. M. Mishra, and R. W. Broerdsen, "Implementation issues in spectrum sensing for cognitive radios," in Proceedings of the 38th Asilomar Conference on Signals, Systems and Computers, pp. 772-776, November 2004.

[24] E. Hossain and V. K. Bhargava, Cognitive Wireless Communication Networks, Springer, 2007.

[25] S. Haykin, "Cognitive radio: brain-empowered wireless communications," IEEE Journal on Selected Areas in Communications, vol. 23, no. 2, pp. 201-220, 2005.

[26] J. Mitola, Cognitive Radio Architecture: The Engineering Foundations of Radio XML, John Wiley \& Sons, 2006.

[27] M. Di Felice, K. R. Chowdhury, and L. Bononi, "Analyzing the potential of cooperative cognitive radio technology on intervehicle communication," in Proceedings of the IFIP Wireless Days (WD '10), October 2010.

[28] M. Manzano, F. Espinosa, A. M. Bravo, D. Garcia, A. Gardel, and I. Bravo, "Medium access control based on a non cooperative cognitive radio for platooning communications," in Proceedings of the IEEE Intelligent Vehicles Symposium, pp. 408-413, 2012. 


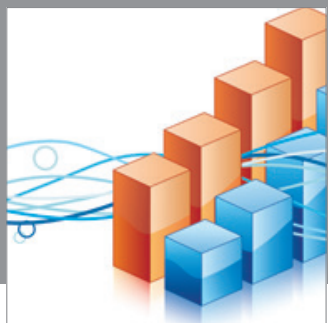

Advances in

Operations Research

mansans

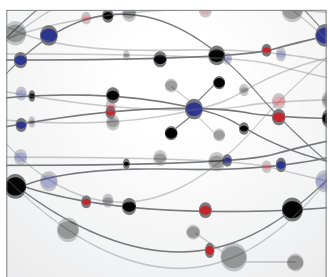

The Scientific World Journal
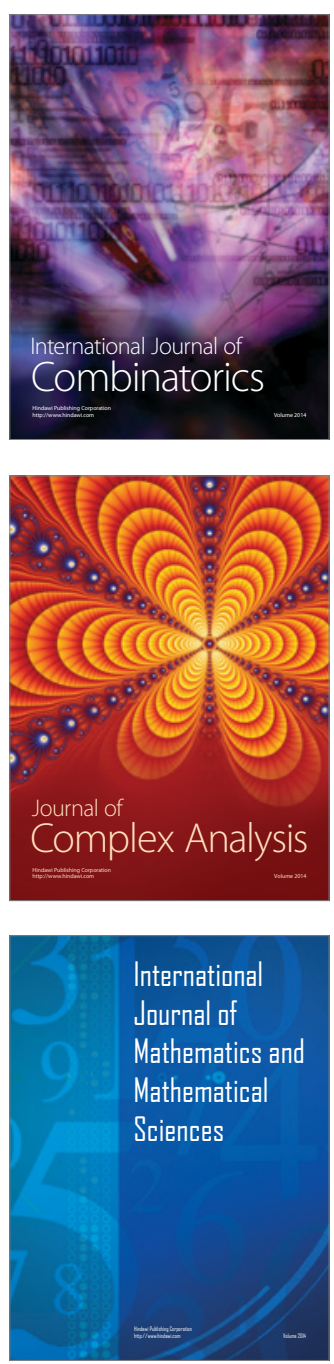
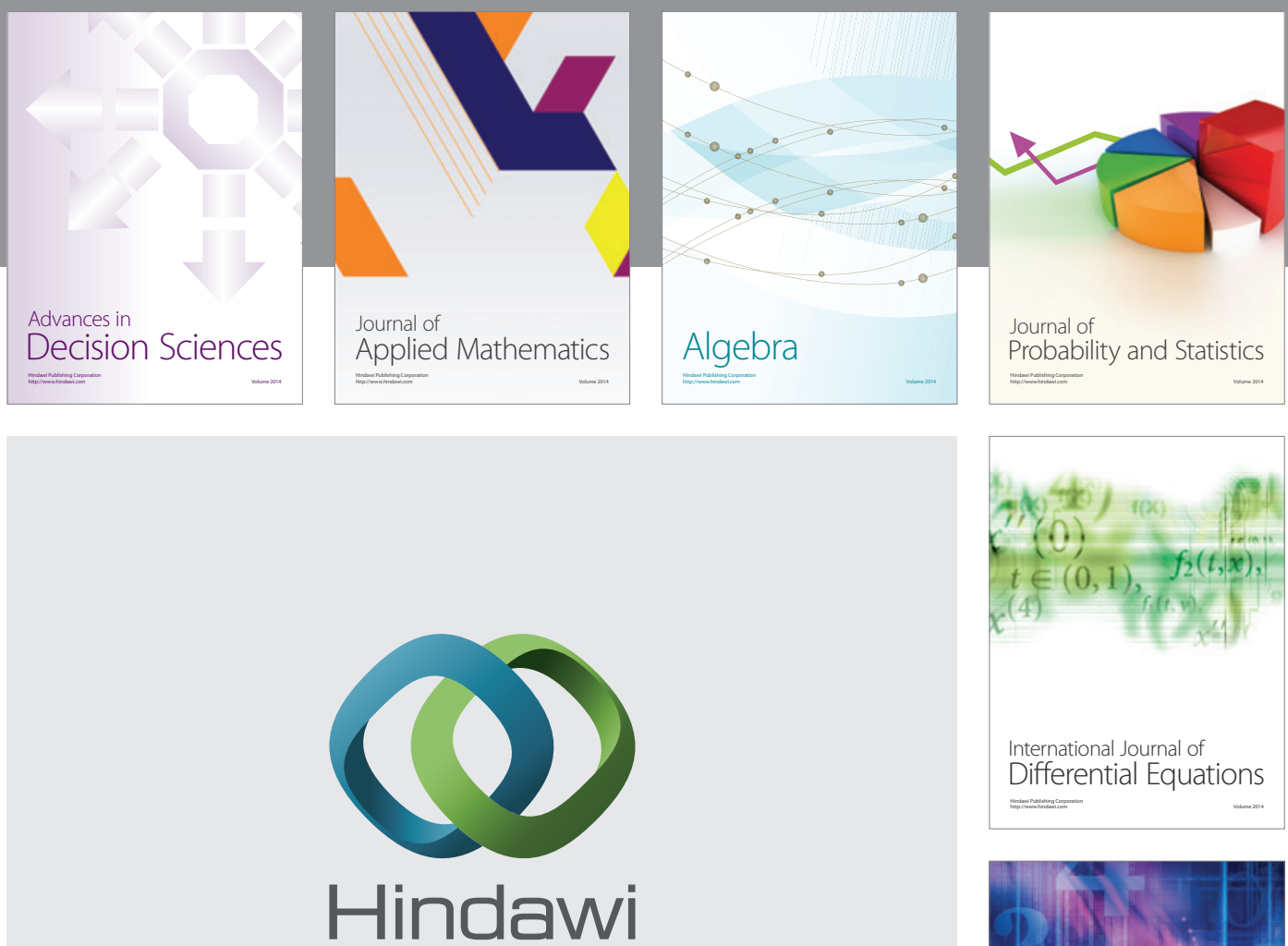

Submit your manuscripts at http://www.hindawi.com
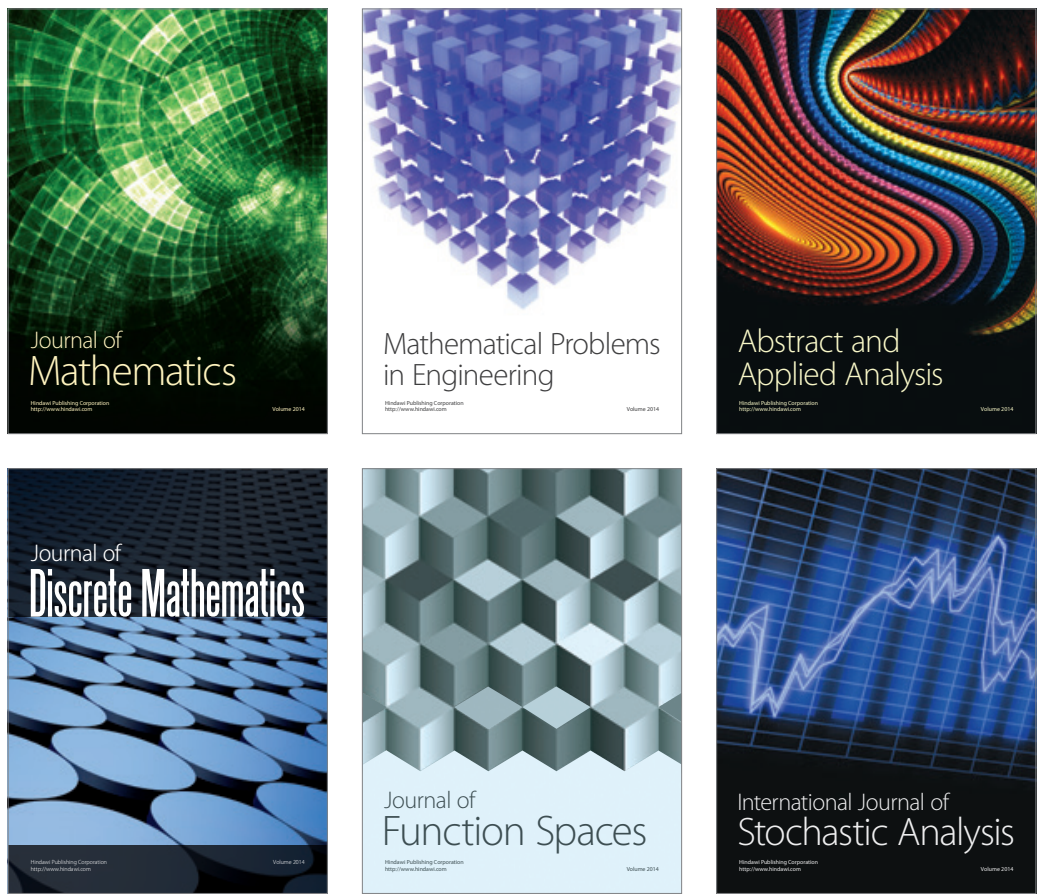

Journal of

Function Spaces

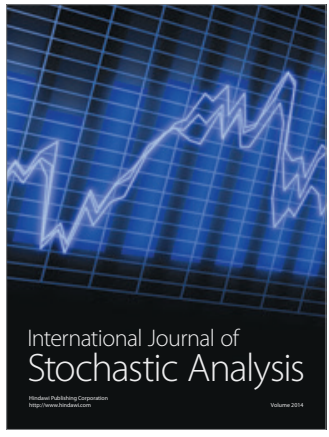

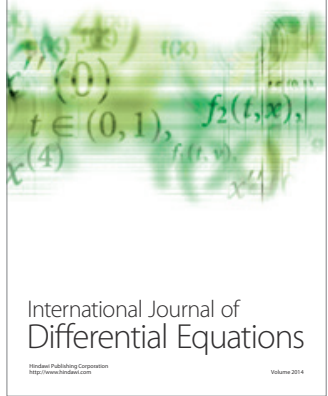
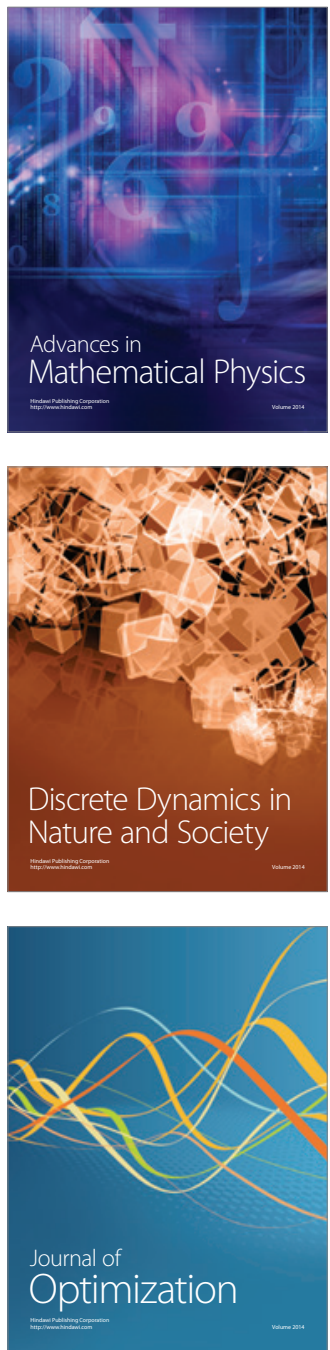